\title{
A New Protocol of Inhalatory Induction of Anaesthesia in Experimental Swine Surgery. A Pilot Study
}

\author{
Mihai Popescu ${ }^{1,2}$, Corina David ${ }^{2}$, Mihail Pautov $^{3}$, Radu Ionescu $^{4}$, Dana Tomescu ${ }^{1,2}$
}

"Carol Davila" University of Medicine and Pharmacy, Department of Anaesthesia and Critical Care, Bucharest, Romania 2Department of Anaesthesia and Critical Care III, Fundeni Clinical Institute, Bucharest, Romania ${ }^{3}$ "Dan Setlacec"Department of General Surgery and Liver Transplantation, Fundeni Clinical Institute, Bucharest, Romania ${ }^{4}$ Istravet Veterinary Clinic, Bucharest, Romania

\section{Corresponding author:}

Corina David, MD

Department of Anaesthesia and Critical Care III, Fundeni Clinical Institute, Bucharest, Romania E-mail: dvd_corina@yahoo.com

\section{ABSTRACT}

Induction of Anaesthesia in swine may prove to be difficult because of the differences in anatomy and physiology. Intubation of large animals is especially difficult due to the length of the snout and the relative difficulty of obtaining adequate relaxation. The present study is aimed to compare two induction techniques for tracheal intubation in pigs. The secondary aim was to assess the effectiveness of a new anaesthetic protocol for experimental surgery. For this, six female pigs underwent two liver surgeries each. Induction of anaesthesia was performed either using intravenous propofol and fentanyl (IV intravenous group) or by using Sevoflurane (inhalatory group). The attending anaesthesiologist was asked to grade the ease of intubation from a scale between 1 and 5. Orotracheal intubation was easier to obtain in the inhalatory group $(p=0.00)$ and a smaller number of attempts were necessary $(p=0.00)$. A lower End-tidal C02 after intubation was observed in the inhalatory group $(p=0.05)$. There were no differences in both systolic $(p=0.11)$ and diastolic $(p=0.72)$ arterial pressure and heart rate $(p=0.16)$ after induction of anaesthesia between the two groups. Maintenance of anaesthesia was made using Sevoflurane and boluses of fentanyl for adequate analgesia while no muscle relaxants were used. Intravenous morphine and ketoprofen were used for postoperative analgesia. In conclusion, volatile induction of anaesthesia is safe and offers a better view of the larynx in large swine, while the proposed anaesthetic protocol provides adequate anaesthesia for the surgeons and assures comfort to the animals.

Key words: experimental surgery, Swine surgery, volatile induction, intubation

\section{INTRODUCTION}

Anaesthesiologists worldwide are getting more and more involved in animal research studies. The development of multidisciplinary teams of practitioners (1), including surgeons, anaesthesiologists, radiologists and pathologists for experimental surgeries in swine offers new challenges to doctors as both pig anatomy and physiology are different (2). 
From the anaesthesiologist perspective, assuring an adequate airway may prove very difficult due to the length of the snout and of the larynx. A laryngeal mask airway may offer some benefits (3) but it is not adequate for long procedures or laparoscopic surgery. Many studies in humans demonstrated that volatile induction of anaesthesia is associated with a higher cardiovascular stability (4) and improved oxygenation (5). On the other hand cost effectiveness is important in funding for research grants and adequate perioperative intensive care must ensure low costs, adequate operating conditions and animal comfort. As so, the present study focuses on the development of an individualized anaesthetic protocol for experimental swine surgery.

\section{METHODS}

Ethical approval for the present study was granted by Fundeni Clinical Institute Ethical Committee, in accordance with principles of the Declaration of Helsinki. We analyzeddata obtained from 6 swine that underwent hepatic surgery. Each swine underwent two surgeries: during the first one cryoelectrolysis and electrolytic electroporation was applied and during the second one ( 24 hours after the previous surgery) a hepatectomy was performed and the pigs were euthanized using a $7.45 \%$ potassium chloride solution (1 $\mathrm{ml} / \mathrm{kg}$ ) and $0.2 \mathrm{mg} / \mathrm{kg}$ iv morphine while under general anaesthesia.

The primary aim of this study was to compare two induction techniques in term of ease ofintubation. The secondary outcomewas to assess the effectiveness of an anaesthetic regimen applied in experimental swine surgery.

Six domesticated female pigs were randomly chosen from a commercial farm based on weight (in order to have between 75 and $100 \mathrm{~kg}$ ). In order to minimize environmental stress, the animals were moved and housed at Fundeni Clinical Insitute Centre for Excelence in Translational Medicine for at least 24 hours before surgery. The pigs were housed alone in dedicated rooms with a surface of at least $5 \mathrm{~m}^{2}$. The room was heated and humidified appropriately in order to make the animals comfortable. Food was withheld 24 hours before surgery and water before 6 hours.

The following variables were recorded. The swine were weighed and measured (snout length, snout diameter, snout to tail length, snout to vertex, snow to $12^{\text {th }}$ rib). Induction of anesthesia: the swine were equally divided into two groups: in the first group (iv group) an intravenous induction was applied (propofol and fentanyl) and in the second group (inhale group) an inhalation technique using $8 \%$ Sevoflurane was applied. The swine were intubated with cuffed 7.0 endotracheal tube by using a number 4 Miller blade. After induction of anaesthesia an assistant held the pigs' snout opened using two loops made of gauze. The tongue was firmly held with a gauze as the anaesthesiologist carefully introduced the blade from the right lateral side of the snout and advanced it midline until the epiglottis was visible. Further advancement of the blade made visible the entrance to the larynx. The endotracheal tube was advanced through the larynx and after the tip passed the arytenoids the tube was further advanced by rotation for a further 3 to $5 \mathrm{~cm}$ as the larynx in pigs has a much larger length compared to humans (fig. 1 and 2).

The anaesthesiologist was asked to grade the ease of intubation from 1 (most difficult/inability to intubate) to 5 (easiest to intubate). The number of attempts until intubation was successful was also noted. Preoperative vital signs (heart rate and blood pressure) were noted at induction and there after at 5 minutes intervals. For maintenance of anaesthesia we useda Primus Anaesthesia workstation (Drager $^{\circledR}$, Lubeck, Germany) equipped with a sevoflurane vaporizer. Endtidal $\mathrm{CO} 2$ was noted immediately after intubation.

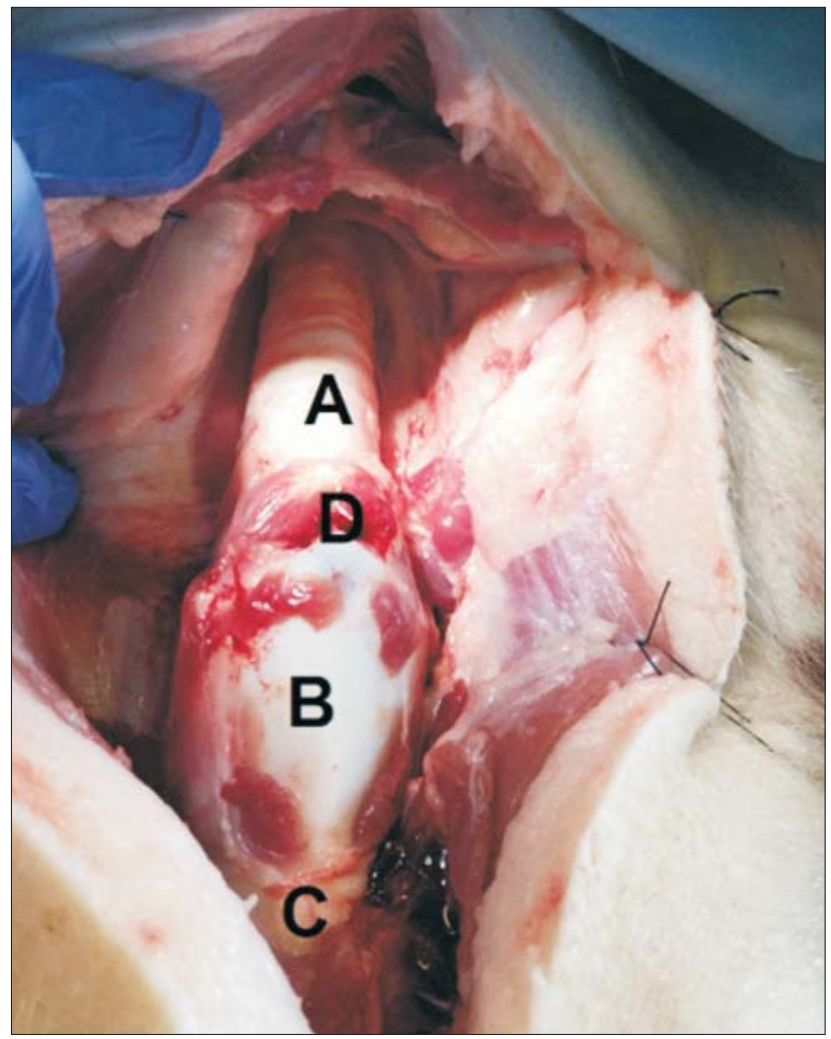

Figure 1 - Dysection of the larynx in pigs.

Legend: A - trachea, B - larynx, C - opening of larynx, $D$ - first tracheal ring 


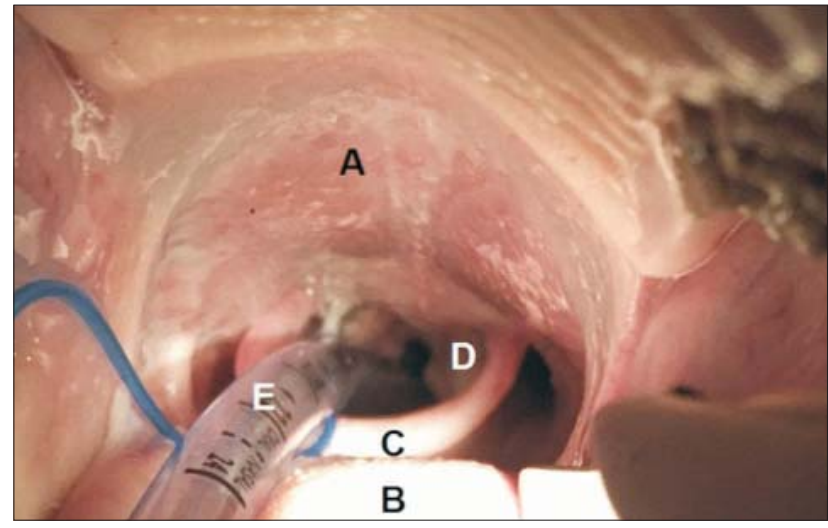

Figure 2 - Tracheal intubation in pigs. View at laryngoscopy. Legend: A - hard palate, B - base of tongue, C - epiglottis, D - cartilagoarytenoidea, $\mathrm{E}$ - endotracheal tube

Intraoperative drug requirements and minimum alveolar concentration (MAC) of inhaled anaesthetic agent were noted. During the postoperative period, the swine were kept in separated, well heated rooms and appropriate analgesia was administered.

Statistical analyses were performed using SPSS 19.0 (SPSS Inc ${ }^{\circledast}$, Chicago, IL, USA). Data are presented as mean \pm standard deviation of the mean or percentage. Ease of intubation and number of attempts were compared between the two groups using Gamma test. Data distribution was examined in order to insure the proper statistical examination. Quantitative data were analyzed with independent samples t-test if normal distributed or Mann-Whitney test if the data did not follow a normal distribution. All p-values are two-tailed. Statistical significance was considered at a p-value < 0.05 .

\section{RESULTS}

\section{Preanaesthesia and induction of anaesthesia}

The anthropometric measures are presented in table 1 . The swine were sedated using intramuscular $0.5 \mathrm{mg} / \mathrm{kg}$ acepromazine and $15 \mathrm{mg} / \mathrm{kg}$ ketamine. Venous access was obtained by catheterization ofone of the auricular veinusing a $20 \mathrm{G}$ peripheral venous catheter. Induction of anaesthesia was then performed with the pig in the ventral position. In the iv group the animals were pre-oxygenated using a tight-fitting snout mask with $100 \%$ oxygen at a flow rate of $12 \mathrm{~L} / \mathrm{min}$ for at least 3 minutes. Intravenous fentanyl $(1 \mathrm{mcg} / \mathrm{kg})$ and propofol $(2 \mathrm{mg} / \mathrm{kg})$ were administered and tracheal intubation attempted.In the inhale group the anaesthetic circuit was primed for 5 minutes using an inspired $8 \%$ sevoflurane concentration. The tight fitting mask
Table 1 - Antropometric parameters

\begin{tabular}{lc}
\hline Anthropometric parameter & Value $( \pm$ SD $)$ \\
\hline weight $(\mathrm{kg})$ & $91.7 \pm 7.5$ \\
\hline snout length $(\mathrm{cm})$ & $20.3 \pm 2.0$ \\
\hline snout diameter $(\mathrm{cm})$ & $33.6 \pm 3.5$ \\
\hline snout to tail length $(\mathrm{cm})$ & $131.0 \pm 2.6$ \\
\hline snout to vertex $(\mathrm{cm})$ & $28.0 \pm 2.0$ \\
\hline snout to $12^{\text {th }}$ rib $(\mathrm{cm})$ & $95.6 \pm 2.5$ \\
\hline
\end{tabular}

was then applied on the pigs snout and the animal was left to breathe spontaneously for several minutes (fig. 3). Intubation was assessed as significantly easier in the inhale group and a smaller number of attempts were made to intubate (table 2).There were no statistical differences in hemodynamic parameters between inhale group and iv group althoug a smaller end-tidal $\mathrm{CO} 2$ was observed in the inhale group probably due to the smaller number of attempts (table 3).

A central venous catheter was placed percutaneously in the internal jugular vein. The needle was inserted 3 to $5 \mathrm{~cm}$ lateral of the midline of the neck with the tip of the needle facing the midline of the sternum. For better understanding, the anatomy of the internal jugular vein is presented in fig. 4.

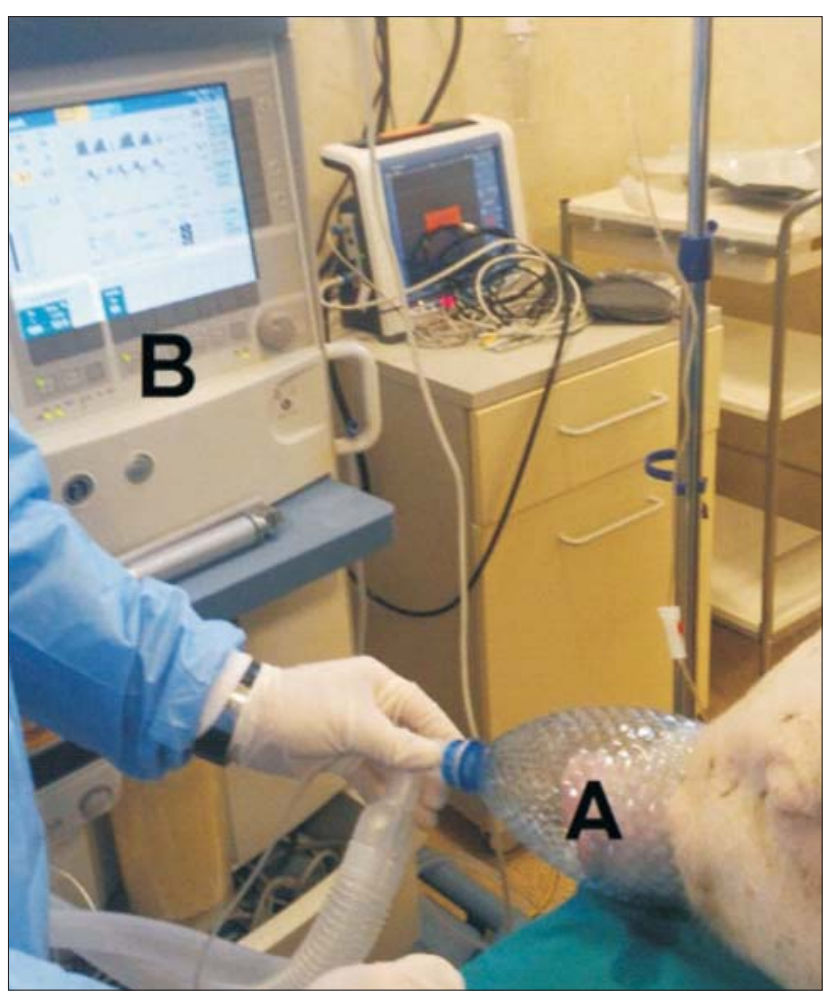

Figure 3 - Volatile induction of anaesthesia in pigs by using a tight fitting mask on the animals' snout $(A)$ coupled to the anaesthesia machine with the inhaled anaesthetic agent turned on at a concentration of $8 \%$ (B) while the animal is breathing spontaneously 
Table 2 - Comparison of difficulty of intubation and number of attempts between iv group and inhale group [data are presented as median (minimum, maximal)]

\begin{tabular}{lccc}
\hline & IV Group & Inhale group & P value \\
\hline Difficulty of intubation (1-5 grade) & $3(2,4)$ & $5(4,5)$ & $\mathbf{0 . 0 0}$ \\
\hline Number of attempts & $3.5(1,5)$ & $1(1,2)$ & $\mathbf{0 . 0 0}$ \\
\hline
\end{tabular}

Table 3 - Haemodynamic parameters compared between groups

\begin{tabular}{|c|c|c|c|c|}
\hline Paramaters & All surgeries $(n=12)$ & Inhale group $(n=6)$ & IV group $(n=6)$ & $P$ value \\
\hline APsyspreind & $139.8 \pm 17.4$ & $145.6 \pm 13.2$ & $134.0 \pm 22.0$ & 0.48 \\
\hline APdiapreind & $75.8 \pm 11.4$ & $82.3 \pm 10.7$ & $69.3 \pm 9.29$ & 0.18 \\
\hline HR preind & $76.8 \pm 13.4$ & $80.0 \pm 13.0$ & $73.6 \pm 15.9$ & 0.62 \\
\hline APsyspostind & $126.5 \pm 38.7$ & $151.3 \pm 38.8$ & $101.6 \pm 18.0$ & 0.11 \\
\hline APdiapostind & $66.0 \pm 13.9$ & $68.3 \pm 10.4$ & $63.6 \pm 7.0$ & 0.72 \\
\hline HR postind & $104.6 \pm 38.3$ & $85.0 \pm 6.24$ & $123.3 \pm 50.4$ & 0.26 \\
\hline EtCO2 & $43.1 \pm 4.3$ & $40.0 \pm 2.64$ & $46.3 \pm 3.05$ & 0.05 \\
\hline
\end{tabular}

Legend: AP - arterial pressure; sys - systolic; dia - diastolic, HR - heart rate, preind - preinduction of anaesthesia, postind- postinduction of anaesthesia, EtCO2 - end tidal carbon dioxide

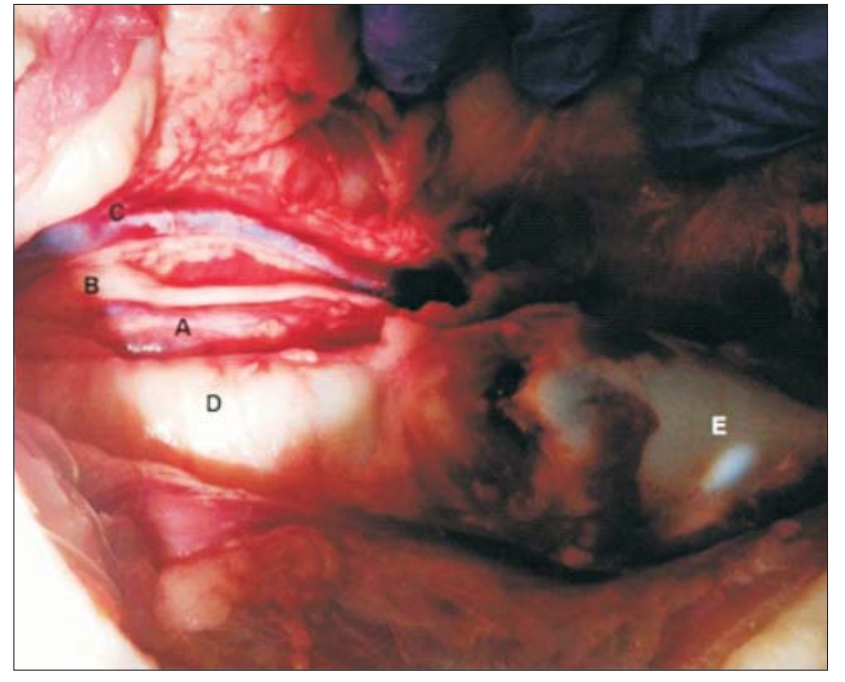

Figure 4 - Great vessels of the neck in pigs. Legend: A - vagus nerve, B - carotid artery, C - internal jugular vein, D - trachea, E - larynx

\section{Maintenance of anaesthesia}

After induction of anaesthesia the pigs were mechanically ventilated in volume-control using a tidal volume of $10 \mathrm{ml} / \mathrm{kg}$, a respiratory rate adjusted (12 to 18 breaths per minute) to maintain normocapnia and a PEEP of $3 \mathrm{mmHg}$ was applied. The fresh gas flow (80\% oxygen in air) was set to $2 \mathrm{~L} / \mathrm{min}$. Anaesthesia was maintained using sevoflurane set to maintain a MAC between 1.5 and 2.5 (EtSev 1.5-2.5 MAC). Further analgesia was achieved using $0.1 \mathrm{mg}$ bolus doses of fentanyl at the anaesthesiologists discretion. No muscle relaxants were used during surgery. Warm Ringer solution was administered for the entire duration of surgery at a rate of $3 \mathrm{ml} / \mathrm{kg} / \mathrm{h}$. At the end of surgery a bolus dose of $250 \mathrm{ml}$ of $10 \%$ glucose was administered. For postoperative analgesia $1.5 \mathrm{mg} / \mathrm{kg}$ ketoprofen and $0.1 \mathrm{mg} / \mathrm{kg}$ of morphine were administered intravenously at skin closure. At the end of surgery the inhaled anaesthetic was discontinued, the pigs were left in spontaneous breathing mode and the trachea was extubated when spontaneous tidal volume was at least $5 \mathrm{ml} / \mathrm{kg}$ and the respiratory rate at least 12 breaths/min.

\section{Postoperative care}

During postoperative care intravenous $0.1 \mathrm{mg} / \mathrm{kg}$ of morphine and $1 \mathrm{mg} / \mathrm{kg}$ ketoprofen were administered every 8 hours. In addition $2 \mathrm{mg} / \mathrm{kg}$ meperidine was administered intravenously two hours after surgery. The pigs were not allowed to eat but were allowed to drink water during the 24 hours between surgeries.

\section{DISCUSSION}

During experimental swine surgery anaesthesia must provide both comfort and safety of the animal and the desired level of anesthesia and relaxation for the surgical staff. The anaesthetic regimen is of crucial importance especially in cases in which re-intervation is planed and the animals must be safely weaned from mechanical ventilation and adequate conform and analgesia provided during the postoperative period. The anaesthetic technique proposed is feasible for the purposes mentioned.

Intubation in swine is hard to achieve especially in large animals (6). Studies (7) have already shown that the 
ventrodorsal position is recommended for orotracheal intubation. Nevertheless, induction techniques vary from center to center (8). To the authors knowledge few papers were pubșished worldwide on this topic. Because of the small number of animals included, the present study lacks statistical power, the highly significant results that favor inhalational induction demonstrates the potential benefits in terms of ease of intubation and hemodynamic stability. Further investigations are needed in order to reach definitive results.

Although some studies favor intravenous maintenance of anaesthesia $(9,10)$, in terms of safety and hemodynamic stability, one must remember that new inhaled anaesthetics such as sevoflurane offer both cardioprotection and ease of administration (11,12). A balanced approach (13) using both intravenous and inhaled agents has been proposed and probably offers the potential benefits of both techniques. Our protocol showed similar results.

As founding for experimental research and surgery is hard to find, cost effectiveness must be addressed. Although most studies $(14,15)$ come from analysis of human surgeries the same principle applies and inhalational maintenance of anaesthesia is more appropriate from a financial point of view.

Postopreative anaesthesia care is crucial from both an ethical point of view and for the animals' safety. If reintervention is planned, adequate analgesia, comfort and hydration are essential. Both transdermal and intravenous opioids have been successfully used for pain relief (16). In our opinion, the combination of morphine and non-steroid anti-inflammatory drugs offers successful pain relief at small costs. The effects of hypothermia on different organs and systems are well documented (17). Intraoperative warming using forced heated air is strongly recommended as well as warming the postoperative facility to at least 23-24 degrees Celsius. Postoperative shivering was successfully treated using meperidine.

\section{CONCLUSION}

In conclusion, our proposed protocol for anaesthesia (table 4) in hepatic surgery in swine is feasible and offers both cost-effectiveness and safety. Inhalation induction of anaesthesia may be superior to classic intravenous protocols but a larger randomized control trial is required in order to reach definitive results.

Table 4 - Anaesthetic protocol in experimental swine surgery

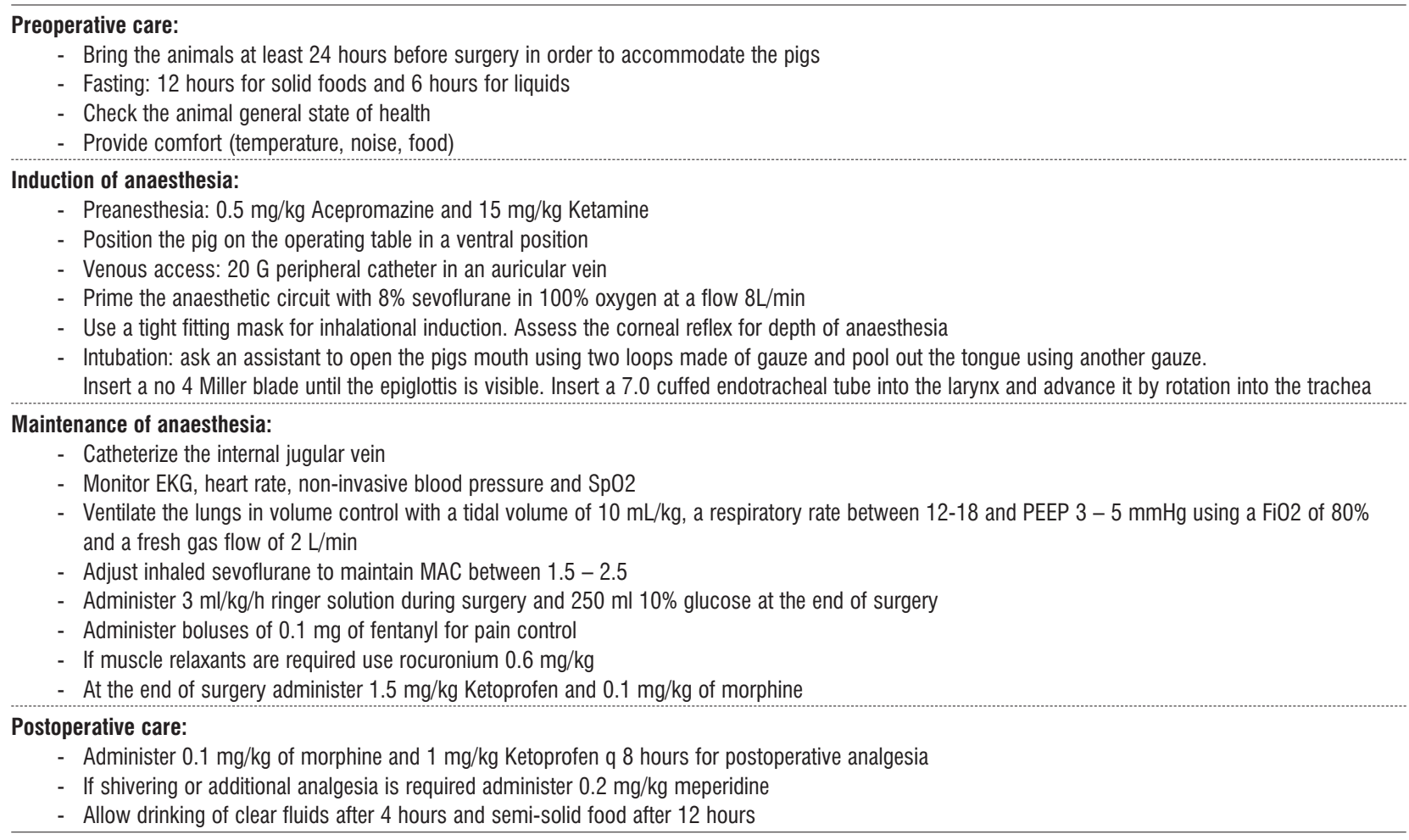




\section{Conflict of interest}

The authors have no conflict of interest to declare.

\section{REFERENCES}

1. Swindle MM, Smith AC. Best practices for performing experimental surgery in swine. J Invest Surg. 2013 Apr;26(2):63-71.

2. Lossi L, D'Angelo L, De Girolamo P, Merighi A. Anatomical features for an adequatechoice of experimental animal model in biomedicine: II. Small laboratory rodents, rabbit, andpig. Ann Anat. 2016 Mar;204:1128.

3. Fulkerson PJ, Gustafson SB. Use of laryngeal maskair way compared to endotracheal tube with positive-pressure ventilation in anesthetized swine. Vet Anaesth Analg. 2007 Jul;34(4):284-8.

4. Siampalioti A, Karavias D, Zotou A, Kalfarentzos F, Filos K. Anesthesia management for the super obese: is sevoflurane superior to propofol as a sole anesthetic agent? A double-blind randomized controlled trial. EurRev Med Pharmacol Sci. 2015 Jul;19(13):2493-500.

5. Kondo $\mathrm{Y}$, Hirose N, Maeda T, Suzuki T, Yoshino A, Katayama $Y$. Changes in Cerebral Blood Flowand Oxygenation During Induction of General Anesthesia with Sevoflurane Versus Propofol. Adv Exp Med Biol. 2016;876:479-84.

6. Chum H, Pacharinsak C. Endotracheal intubation in swine. Lab Anim (NY). 2012 Nov;41(11):309-11.

7. Theisen MM, Maas M, Hartlage MA, Ploner F, Niehues SM, Van Aken $H K$, et al. Ventral recumbencyis crucial for fast and safe orotracheal intubation in laboratory swine. Lab Anim. 2009 Jan;43(1):96-101.

8. Kaiser GM, Heuer MM, Frühauf NR, Kühne CA, Broelsch CE. Genera handling and anesthesia for experimental surgery in pigs. J Surg Res. 2006 Jan;130(1):73-9.
9. Kurita T, Morita K, Kazama T, Sato S. Comparison of isoflurane and propofol-fentanyl anaesthesia in a swine model of asphyxia. $\mathrm{Br} \mathrm{J}$ Anaesth. 2003 Dec;91(6):871-7.

10. Kaiser GM, Frühauf NR, Zhang $\mathrm{H}$, Westermann $\mathrm{S}$, Bolle I, Oldhafer KJ, Broelsch CE. Intravenous infusion anesthesia with PropofolMidazolam-fentanyl for experimental surgery in swine. J Invest Surg. 2003 Nov-Dec;16(6):353-7.

11. Otsuki DA, Fantoni DT, Holms C, Auler JO Jr. Minimum alveolar concentrations and hemodynamic effects of two different preparations of sevoflurane in pigs. Clinics (Sao Paulo). 2010 May;65(5): $531-7$.

12. Shiomi M, Miyamae M, Takemura G, Kaneda K, Inamura Y, Onishi A, et al. Sevoflurane induces cardioprotection through reactive oxygenspecies-mediated upregulation of autophagy in isolated guinea pighearts. J Anesth. 2014 Aug;28(4):593-600.

13. Calzetta L, Rossi P, Bove P, Alfonsi P, Bonizzi L, Roncada P, et al. A Noveland Effective Balanced Intravenous-Inhalant Anaesthetic Protocol in Swine by Using Unrestricted Drugs. Exp Anim. 2014;63 (4):423-33.

14. Kumar G, Stendall C, Mistry R, Gurusamy K, Walker D. A comparison of total intravenous anaesthesia using propofol with sevoflurane or desflurane in ambulatorysurgery: systematic review and metaanalysis. Anaesthesia. 2014 Oct;69(10):1138-50.

15. Singh $Y$, Singh AP, Jain G, Yadav G, Singh DK. Comparative evaluation of cost effectiveness and recovery profile between propofol and sevoflurane in laparoscopic cholecystectomy. Anesth Essays Res. 2015 May-Aug; 9(2): 155-160.

16. Harvey-Clark CJ, Gilespie K, Riggs KW. Transdermal fentanyl compared with parenteral buprenorphine in post-surgicalpain in swine: a case study. Lab Anim. 2000 0ct;34(4):386-98.

17. Dent BT, Stevens KA, Clymer JW. Forced-Air Warming Provides Better Control of Body Temperature in Porcine Surgical Patients. Vet. Sci. 2016, 3, 22; doi:10.3390/vetsci3030022 